SHORT REPORT

\title{
Evaluation of swab transport systems against a published standard
}

\section{R P Human, G A Jones}

J Clin Pathol 2004;57:762-763. doi: 10.1136/icp.2004.016725

Background: Before the publication of the M40-A standard by the United States National Committee for Clinical Laboratory Standards (NCCLS), no quality control data for swab transport systems (STSs) had been available.

Aims: To compare three commercially available STSs against the published standard to ascertain bacterial survival after a set holding time.

Method: Charcoal and non-charcoal containing swabs were inoculated with standard volumes and numbers of a range of bacteria commonly isolated from clinical material. Bacterial counts were taken at time zero, six, 24 , and 48 hours, with the results being compared against the NCCLS standard.

Results: The standard clearly differentiated between the three STSs tested, with one product being superior to the other two products in providing better survival.

Conclusion: The standard now provides manufacturers and users of STSs with meaningful data to provide, purchase, and compare an STS for routine clinical use.

$\mathrm{T}$ he potential for delayed transportation to the laboratory is now more likely with the centralisation of laboratory services, use of evening surgeries, and community based clinics. The Department of Health pathology modernisation programme ${ }^{1}$ outlines the strategy to modernise and rationalise pathology. It underpins the delivery of a fast, accurate diagnostic system, which seeks to develop standardised large scale processes, infrastructure consolidation, and patient centred testing by the formation of pathology networks. It is imperative that swab transport systems (STSs) provide optimised preservation of bacteria to produce an accurate diagnosis for the timely treatment of bacterial infection.
Before the production of the United States National Committee for Clinical Laboratory Standards (NCCLS) M40-A standard ${ }^{2}$ there had been no recognised standard for the performance of STSs. The standard now provides a method of quality control testing, together with acceptance criteria not only for viability but also for overgrowth of bacteria.

"The potential for delayed transportation to the laboratory is now more likely with the centralisation of laboratory services, use of evening surgeries, and community based clinics"

In the absence of a standard procedure for determining the effectiveness of STSs, previous papers on this subject have only been able to provide comparative data. The new standard resolves this by defining whether a product is acceptable in terms of bacterial survival.

\section{METHODS}

The STSs tested were both non-charcoal and charcoal containing and were supplied by Bibby Sterilin (M40 product) manufactured by Copan Italia, Brescia, Italy; Medical Wire and Equipment (MW170/1) Corsham, UK; and Technical Service Consultants (TS5-17/18) Heywood, Lancashire, UK.

Bacterial strains used for viability studies were Haemophilus influenzae (ATCC 10211), Neisseria gonorrhoeae (ATCC 43069), Streptococcus pneumoniae (ATCC 6305), Prevotella melaninogenica (ATCC 25845), and Peptostreptococcus anaerobius (ATCC 27337).

Abbreviations: CFU, colony forming units; NCCLS, National Committee for Clinical Laboratory Standards; STS, swab transport system

Table 1 Comparison of viability studies

\begin{tabular}{|c|c|c|c|c|c|c|c|c|}
\hline Supplier & Transport medium & $\begin{array}{l}\text { Holding } \\
\text { temperature }\left({ }^{\circ} \mathrm{C}\right)\end{array}$ & $\begin{array}{l}\text { Viability } \\
\text { HI }\end{array}$ & GC & PC & Pepto & Prev & $\begin{array}{l}\text { Overgrowth } \\
\text { Pseu }\end{array}$ \\
\hline \multirow[t]{4}{*}{ Sterilin } & Charcoal & $4-8$ & $\checkmark$ & $\checkmark$ & $\checkmark$ & $\checkmark$ & $\checkmark$ & $\checkmark$ \\
\hline & & $20-25$ & $\checkmark$ & $\checkmark$ & $\checkmark$ & $\checkmark$ & $x$ & \\
\hline & Plain & $4-8$ & $\checkmark$ & $\checkmark$ & $\checkmark$ & $\checkmark$ & $\checkmark$ & $\checkmark$ \\
\hline & & $20-25$ & $\checkmark$ & $\checkmark$ & $\checkmark$ & $\checkmark$ & $\checkmark$ & \\
\hline \multirow[t]{4}{*}{ MWE } & Charcoal & $4-8$ & $x$ & $x$ & $\checkmark$ & $\checkmark$ & $x$ & $\checkmark$ \\
\hline & & $20-25$ & $x$ & $x$ & $\checkmark$ & $x$ & $x$ & \\
\hline & Plain & $4-8$ & $x$ & $\checkmark$ & $\checkmark$ & $\checkmark$ & $\checkmark$ & $\checkmark$ \\
\hline & & $20-25$ & $x$ & $x$ & $x$ & $x$ & $x$ & \\
\hline \multirow[t]{4}{*}{ TSC } & Charcoal & $4-8$ & $x$ & $x$ & $\checkmark$ & $\checkmark$ & $x$ & $\checkmark$ \\
\hline & & $20-25$ & $x$ & $x$ & $x$ & $x$ & $x$ & \\
\hline & Plain & $4-8$ & $x$ & $x$ & $\checkmark$ & $x$ & $x$ & $\checkmark$ \\
\hline & & $20-25$ & $x$ & $x$ & $x$ & $x$ & $x$ & \\
\hline
\end{tabular}

$\checkmark$, acceptable; $X$, unacceptable.

GC, Neisseria gonorrhoeae; HI, Haemophilus influenzae; MWE, Medical Wire and Equipment; PC, Streptococcus pneumoniae; Pepto, Peptostreptococcus anaerobius; Prev, Prevotella melaninogenica; Pseu, Pseudomonas aeruginosa; TSC, Technical Service Consultants. 


\section{Take home messages}

- Bacterial survival is dependent upon the manufacturer's product

- Charcoal containing media provide increased survival

- Storage at $4-8^{\circ} \mathrm{C}$ before and during transport to the laboratory increases the survival rates

- The NCCLS standard now provides clear guidance on the quality control of transport systems

For overgrowth studies Pseudomonas aeruginosa (ATCC BAA427) was used.

Strains were subsequently cultured on plates containing $5 \%$ horse blood Columbia agar for $S$ pneumoniae and $P$ s aeruginosa, 5\% horse blood fastidious anaerobic agar for $P$ melaninogenica and $P$ anaerobius, and 5\% horse blood Columbia chocolate agar for $N$ gonorrhoeae and $H$ influenzae. All plates were incubated at $37^{\circ} \mathrm{C}$ in either $\mathrm{CO}_{2}$ or anaerobic atmospheres as appropriate.

For viability studies, inocula were prepared in $0.85 \%$ saline $(\mathrm{pH} 6.8-7.2)$ at a concentration of approximately $1.5 \times 10^{8}$ colony forming units (CFU; equivalent to 0.5 McFarland standard) from a 18-24 hour growth of the organism. From this dilution, final working dilutions were made ranging from $1.5 \times 10^{6}$ to $1.5 \times 10^{4} \mathrm{CFU} / \mathrm{ml}$. A $100 \mu \mathrm{l}$ aliquot of each of these three dilutions was used to inoculate the swabs. For overgrowth studies one dilution of $1.5 \times 10^{3}$ was used.

Although swabs were plated at time zero, six, 24 , and 48 hours after storage at $4-8^{\circ} \mathrm{C}$ and ambient temperature (20$25^{\circ} \mathrm{C}$ ), the standard requires a holding time of 24 hours for $N$ gonorrhoeae and 48 hours for the other species.

Inoculated swabs were rolled over the entire surface of the medium, as stipulated by the standard, and colonies were counted after incubation for $24 / 48$ hours at $37^{\circ} \mathrm{C}$. To ensure consistency, all swabs were tested in triplicate at all dilutions and storage temperatures.

\section{RESULTS}

Colonies were counted at time zero, six, 24, and 48 hours and compared against the time zero count.

The standard clearly states acceptance criteria for recovery as follows:
- For viability studies-there shall be $\geqslant 5$ CFU following the specified holding time from the specific dilution that yielded zero time plate counts closest to $300 \mathrm{CFU}$.

- For overgrowth studies-there shall be no more than 1 log increase in CFU between the zero time count and the counts of the swabs that were held.

Table 1 shows the results as acceptable or unacceptable depending on whether the standard was met.

\section{DISCUSSION}

Our study demonstrated a divergence in the performance of the STSs tested, with the Bibby Sterilin product being the only one capable of meeting the standard.

The performance of the STSs, which is dependent on the manufacturer, could have clinical relevance-for example, with the lack of isolation of organisms such as $N$ gonorrhoeae.

The rigorous M40-A standard gives manufacturers a goal to provide a product that will best serve the needs of the patient by assuring bacterial survival during transportation. Laboratories now have guidance to select the best STS for viability, which should not be compromised by cost.

It would now seem prudent with the publication of the NCCLS standard that European countries should adopt this standard to ensure conformity with manufactures' performances.

Results clearly demonstrate that swabs held at $4-8^{\circ} \mathrm{C}$ provide better viability than those held at ambient temperature; users should be encouraged to place swabs in the refrigerator if delay in sending to the laboratory is likely.

\section{Authors' affiliations \\ R P Human, G A Jones, Microbiology Laboratory, Derriford Hospital, Plymouth, Devon PL6 8DH, UK}

Correspondence to: Mr R P Human, Microbiology Laboratory, Derriford Hospital, Plymouth, Devon PL6 8DH, UK; robert.human@phnt.swest.nhs. uk

Accepted for publication 9 February 2004

\section{REFERENCES}

1 Modernisation of Pathology Services. Department of Health HSC 1999/170. London: Department of Health.

2 NCCLS. Quality Control of Microbiological Transport Systems; Approved Standard. NCCLS document M40-A (ISBN 1-56238-520-8). Pennsylvania, USA: NCCLS. 